\title{
Editorial
}

\section{Recherche sur les services de santé dans le secteur ambulatoire}

Jean Martin'

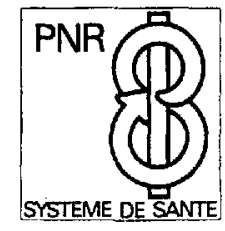

L'équipe de recherche du projet «Quantification et qualification des prestations de soins ambulatoires» du Programme national de recherche N" 8 (PNR 8) est heureuse de présenter dans ce numéro de Médecine sociale et préventive un échantillon des analyses faites à partir de données recueillies en 1980/1981 dans les cantons de Vaud et Fribourg (et, pour les physiothérapeutes, dans l'ensemble de la Suisse romande). Les communications ci-après donnent, pensons-nous, une bonne idée de la variété et de la richesse des résultats que peut fournir la recherche sur les services de santé (health services research). Nous aimerions les introduire par quelques commentaires.

Les besoins d'une telle recherche (relativement nouvelle) ne nécessitent pas de longs développements. Rappelons simplement que les thèmes des Programmes nationaux tels que le PNR 8 sont fixés par le Conseil fédéral lui-même, sur la base de son appréciation des questions prioritaires dans la vie du pays. Dans le cas particulier, l'observateur ne peut que se rallier au choix fait: beaucoup de voix, en Suisse comme dans l'ensemble du monde industrialisé, se sont manifestées au cours des années récentes pour demander plus de bases de décision chiffrées en matière médico-sanitaire. Bases de décision qui aident les responsables à prendre les options nécessaires quant à une politique de santé, au niveau des vingt-six cantons, responsables principaux dans ce domaine, de la Confédération, d'institutions de formation, d'associations professionnelles, etc.

Pour ce qui est des circonstances de la recherche et des contraintes qu'elle peut rencontrer, il faut considérer plusieurs conditions particulières:

- Il s'agit de saisir la réalité de prestations effectives, d'actes (parfois d'attitudes et d'opinions); il ne saurait y avoir simulation (tout au moins dans le premier temps de la collecte de données en vue d'une description factuelle de ce qui se passe, préalable à toute démarche prospective ultérieure).

- Il s'agit (souvent) de saisir une réalité dans le domaine très privé du colloque singulier entre un traitant/ soignant et un patient, domaine protégé par un secret qui garde toute son importance.

- Il s'agit de saisir cette réalité auprès de professionnels très marqués par l'obligation de confidentialité qu'on vient d'évoquer et qui parfois, sans qu'il y ait forcément intention précise, tendent à l'étendre à tout ce qui les concerne. De plus, ils sont souvent suroccupés et n'ont guère été exposés jusqu'ici, ni dans leur formation, ni dans leur pratique, à la recherche sur les services de santé. C'est dire que, quand plus de $50 \%$ du corps médical renvoie rempli un questionnaire comme celui de notre étude structurelle, on doit plus se féliciter de ce qu'une bonne moitié réponde plutôt qu'être déçu que certains ne réagissent pas.

- Un point important nous parait être le suivant: l'incrédulité (implicite plus souvent qu'explicite), parfois la méfiance, vis-à-vis de l'étude statistique d'actes en rapport avec les soins aux malades. Même dans des catégories professionnelles qui ont bénéficié de formations longues comportant un enseignement considérable de sciences naturelles et de mathématiques, on a parfois de la peine à admettre que le fait d'apprécier un profil moyen (du prestataire ou de sa pratique), une statistique de ce qui se passe au cabinet ou dans l'activité au domicile des patients, puisse être approprié et, surtout, utile. "Mais il n'y a que des cas particuliers, toutes les situations sont différentes... Comment voulez-vous qu'une étude d'un échantillon de patients, d'une ou deux semaines d'activité, soit représentative d'un labeur toujours changeant qui porte sur des années?» Il faut répéter que, même s'il y a lieu de rester critique vis-à-vis des statistiques, elles seules permettent d'approcher plus précisément (et plus globalement aussi) une pratique dont la description sinon reste impressionniste.

Requérant responsable du Projet PNR 8 «Soins ambulatoires», médecin cantonal adjoint, Cité-Devant 11, 1005 Lausanne. 
- Enfin, il n'est pas utile de taire que, en tout bien tout honneur, le système de santé représente un secteur de grande importance économique et que des enjeux majeurs y sont en discussion. Même dans une recherche qui (comme la nôtre) n'inclut pas d'éléments financiers, il apparaît probable que certains de nos interlocuteurs se sont posé des questions, voire ont eu des craintes à cet égard.

Cela étant, il reste que les décisions qui doivent être prises quant au système de santé, à son fonctionnement, à son financement, le seront mieux sur la base de faits que sur celle de discussions théoriques ou de batailles d'intérêts. (Etant entendu par ailleurs que ce ne sont pas les résultats de la seule recherche scientifique qui peuvent déterminer ce qui est décidé - s'agissant d'options au niveau d'une collectivité, elles restent en dernière analyse du ressort du politique.)

Notre équipe a d'une part, d'une manière ponctuelle, requis des informations des institutions œuvrant dans le secteur ambulatoire, du corps des médecins praticiens et de celui des physiothérapeutes. D'autre part, elle a demandé une collaboration plus intensive, sur une période d'une ou deux semaines, aux infirmières de santé publique et aux médecins des cantons de Vaud et Fribourg.

Notre bonne fortune a été de bénéficier de la collaboration active des associations professionnelles et des responsables des organismes intéressés. Nous avons fait notre possible pour informer complètement les participants potentiels sur ce qu'étaient nos buts et nos besoins. Nous nous sommes engagés à leur fournir le «feedback» qu'ils étaient en droit d'attendre et nous sommes efforcés, dans cette optique, de mettre rapidement des résultats à leur disposition, par l'intermédiaire de journaux de large audience (même si nous aurions aimé faire encore plus vite). A ce propos, qu'il soit permis une fois encore de remercier tous ceux qui, par leur intérêt, leur bienveillance et leur collaboration effective, ont permis la réalisation de nos travaux.

Au-delà de la collecte des données (phase fondamentale qui s'est déroulée comme prévu, dans des conditions qui paraissent garantes de leur qualité et dans le cadre financier alloué par le Fonds national), un temps et un engagement importants ont été nécessaires pour mettre à profit l'informatique et «sortir» des tableaux, étudier les résultats obtenus, les commenter, les comparer à ceux d'autres études. Dans la mesure où notre équipe n'était pas partie intégrante d'une institution dont la recherche est une mission principale, nous avons fait l'expérience (inquiétante), pendant quelques mois, de savoir que les dossiers informatiques contenaient de quoi procéder à des analyses de grand intérêt (qui se sont souvent révélées, quant à nous, passionnantes) et de ne pas être matériellement en mesure de les faire fructifier. Heureusement, et grâce entre autres à l'intérêt de nos employeurs respectifs, les choses ont évolué à satisfaction.

Quelques mots encore sur les contributions constituant ce numéro:

- Trois d'entre elles (études structurelles - «anatomie» du système) sont des apports à une meilleure connaissance des caractéristiques de groupes professionnels du secteur ambulatoire. Comme le lecteur le remarquera, elles incluent peu de discussions critiques ou de jugements. Il convient d'abord, nous semble-t-il, de cerner la réalité. (De plus, pour ce qui est de la comparer avec d'autres, cela est rendu difficile par la rareté d'études similaires.)

- Les suivantes (études fonctionnelles - «physiologie» du système) illustrent la «versatilité» - diraient les Anglo-Saxons - des analyses que permet l'utilisation d'instruments tels que celui du National Ambulatory Medical Care Survey américain, que nous avons adapté à notre situation pour l'enquête au cabinet des médecins et dont nous nous sommes inspirés quant au principe pour développer une grille spécifique à l'activité des infirmières de santé publique.

La recherche sur les services de santé est en Suisse à ses débuts encore. Elle se développe dans le contexte socio-professionnel, économique et politique qui est le nôtre. Sans qu'on doive s'attendre à une explosion des activités à cet égard, elles prendront certainement plus d'ampleur dans les années qui viennent. Et on peut déjà dire que le Programme national de recherche $\mathrm{N}^{\circ} 8$ aura apporté la contribution facilitatrice que ses promoteurs souhaitaient. 
Il apparaît aussi que les groupes professionnels et institutionnels intéressés sont prêts, s'ils sont impliqués de manière appropriée aux travaux, à y donner leur indispensable collaboration. Cet état de choses devrait permettre de répondre aux demandes faites dans ce domaine (voir bibliographie succincte ci-dessous) et de mettre à disposition des partenaires concernés les bases factuelles dont ils ont besoin à bref délai.

\section{Quelques références récentes}

sur les besoins et la mise en ceuvre de recherche

sur les services de santé en Suisse

- Abelin, Th., Infrastrukturprobleme der sozial- und präventivmedizinischen Forschung in der Schweiz, Sozial- und Präventivmedizin 24, 220-221 (1979).

- Aebi, H., und Frey, $U$. (Hrsg.), Schweizerische Gesundheitspolitik heute und morgen (Bern, Verlag Hans Huber, 1977).

- Bruppacher, R., Forschung im Dienste des Gesundheitswesens. Patient: Gesundheitswesen? Jahrbuch der Neuen Helvetischen Gesellschaft/Annuaire de la Nouvelle Société Helvétique, 1980, 109-127 (Bern, Buri Druck, 1980). Voir aussi:

- Bulletin des médecins suisses 62, 1822-1825 (1981).

- Schweizer Journal, 14-15, März 1981.

- Bruppacher, R., et Ritzel, G. (réd.), Efficience en matière de santé publique (numéro consacré au PNR 8), Sozial- und Präventivmedizin 26, 1-97 (1981).

- Gilliand, P., Information + évaluation: conditions d'un sys- tème efficace de santé, Sozial- und Präventivmedizin 26, 12-20 (1981).

- Kocher, G., Der abnehmende Grenznutzen im Gesundheitssystem, Annuaire de la Nouvelle Société Helvétique, 1980, 37-45 (Bern, Buri Druck, 1980).

- Martin, J., Questions prioritaires pour l'optimisation de la médecine en Suisse (Forum Davos, 1977), Bulletin des médecins suisses 58, 406-409 (1977).

- Quo vadis, schweizerisches Gesundheitswesen? Où va la santé publique suisse? (numéro à thème - plusieurs articles), Bulletin des médecins suisses 62, 1741-1850 (1981).

- Tschopp, P., Le Programme national de recherche sur l'économie et l'efficacité du système de santé en Suisse - Objectif scientifique et portée pratique, Sozial- und Präventivmedizin 26, 6-11 (1981).

- Zimmermann, K., NFP 8: Aufruf zur Mitarbeit/PNR 8: Appel à la collaboration de tous, Schweiz. Ärztezeitung/Bulletin des médecins suisses 63, 1337-1338/1475-1476 (1982). 